\title{
Monitoraggio e gestione delle infezioni virali in riceventi pediatrici di trapianto renale
}

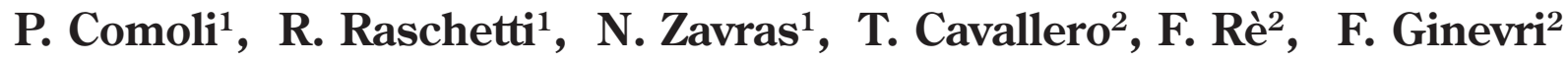 \\ ${ }^{1}$ Oncoematologia Pediatrica, Fondazione IRCCS Policlinico S. Matteo, Pavia \\ ${ }^{2}$ U.O.S. Medicina Clinica e Sperimentale dei Trapianti d'Organo, Istituto G. Gaslini, Genova
}

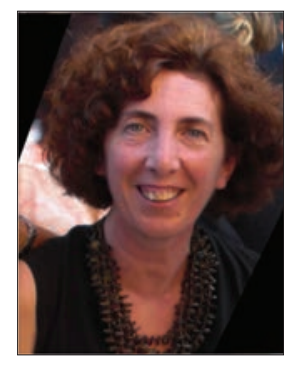

Patrizia Comoli

\section{Introduzione}

Limplementazione delle terapie immunosoppressive ha significativamente migliorato la prognosi del trapianto, facilitando l'attecchimento al di là dei confini posti dall'istoincompatibilità. La sopravvivenza a lungo termine del trapianto e del paziente, tuttavia, è stata ottenuta a scapito di una ridotta sorveglianza immunitaria (1). La mancata attivazione o espansione dell'immunità protettiva si è tradotta, negli ultimi anni, in un significativo aumento del tasso di ospedalizzazione per complicanze infettive (2); inoltre, i pazienti trapiantati presentano con sempre maggior frequenza grave morbilità e mortalità per infezioni da agenti il cui potenziale patogenetico è limitato in soggetti immunocompetenti $(1,3-4)$.

Le infezioni virali sono potenzialmente gravi complicanze del trapianto, in quanto non solo inducono patologie specifiche, ma sono anche gravate da effetti indiretti, quali lo sviluppo di danno d'organo, infezioni opportunistiche e rigetto acuto (1): è stato dunque messo in atto un considerevole sforzo per migliorare il controllo posttrapianto delle infezioni virali. L'applicazione di programmi di monitoraggio virale ha guadagnato considerazione quale utile strumento per raggiungere tale obiettivo, in quanto consente l'identificazione della fase preclinica o precoce della patologia virus-correlata, la valutazione della risposta alla terapia e la caratterizzazione di specifiche coorti di pazienti a rischio $(5,6)$. I recenti progressi della microbiologia molecolare hanno reso possibile la diagnosi di un numero crescente di patogeni virali che si correlano a un importante aumento della morbilità $\mathrm{e}$ della mortalità. É chiaro che un monitoraggio sistematico di tutte le infezioni virali identificabili tramite dei test diagnostici non è fattibile, mentre sarebbe possibile, e dovrebbe essere attuata in ogni Centro, la selezione delle infezioni virali che possono beneficiare di un programma di sorveglianza sulla base della prevalenza, della gravità e della disponibilità di interventi terapeutici. Devono inoltre essere presi in considerazione aspetti relativi all'epidemiologia locale e alle caratteristiche della coorte da monitorare, quali regimi immunosoppressivi e percentuale di individui virus-sieronegativi.

Il miglioramento della gestione dell'infezione virale dell'ospite immunocompromesso è in parte attribuibile ai progressi avvenuti nell'ambito della virologia diagnostica. Linizio della replicazione virale dopo il trapianto dipende dall'esposizione a un dato patogeno, in assenza di una risposta immunitaria protettiva a causa dell'immunosoppressione: per questo motivo, il parametro che è stato universalmente scelto per monitorare le infezioni è la carica virale. Lo sviluppo e l'applicazione di test sensibili, specifici e affidabili, che consentano la quantificazione della carica virale, si sono rivelati fondamentali per aumentare l'utilità clinica del monitoraggio virale (6-8). Inoltre, giacché il controllo delle infezioni dipende in ultima analisi dal ripristino di una risposta immunitaria protettiva, la valutazione di un'immunità virus specifica ha consentito sia un'ulteriore caratterizzazione di sottogruppi di pazienti ad alto rischio di sviluppare una malattia $(9,10)$, che lo sviluppo di strategie terapeutiche basate sulla somministrazione di linfociti $T$ antigene-specifici espansi in vitro dal pool di linfociti della memoria (10, 11). Infine, lo sviluppo e l'applicazione di nuovi agenti te- 
rapeutici anti-virali ha contribuito notevolmente alla gestione ottimale delle infezioni dopo il trapianto $(12,13)$.

\section{Monitoraggio virale}

Il numero delle infezioni virali rilevanti è in costante aumento. Sulla base della prevalenza, gravità, disponibilità di test diagnostici e possibilità di intervento terapeutico, $i$ virus che sono attualmente monitorati con maggiore frequenza nei Centri di trapianto renale, eccettuati i virus dell'epatite e il citomegalovirus, includono il polyomavirus BK (BKV) e il virus di Epstein-Barr (EBV).

\section{Polyomavirus BK}

La nefropatia polyomavirus BK (BKV)-associata (BKVAN) è attualmente la più importante causa infettiva di disfunzione e perdita del trapianto renale (14-17). Il BKV è un polyomavirus umano con una sieroprevalenza superiore al $90 \%$ nella popolazione adulta. La trasmissione del BKV avviene durante l'infanzia. I siti di latenza o di replicazione virale dopo l'infezione primaria sono rappresentati dalle cellule dell'epitelio tubulare renale e dall'urotelio (18). La patologia da BKV è principalmente associata a uno stato di immunodeficienza, anche se la riattivazione e una limitata replicazione virale si possono osservare in una piccola frazione di individui immunocompetenti (18).

La BKVAN rappresenta una complicanza legata a un'elevata replicazione virale nel rene trapiantato $(8,19-21)$. La prevalenza della BKVAN va dall' $1 \%$ al $10 \%$, e circa i tre quarti dei casi si verificano nei primi anni post-trapianto $(14,22)$. La prevalenza nei pazienti pediatrici è simile a quella osservata negli adulti $(22,23)$. La perdita del trapianto renale si verifica dal $10 \% \mathrm{a}<80 \%$ dei casi $(14,15)$, e il più alto tasso di perdita si osserva nei casi con diagnosi tardiva o con fallimento del trattamento $(15-17,21)$. Ad oggi, l'intervento terapeutico di scelta è la riduzione e/o la sostituzione della terapia immunosoppressiva di mantenimento, poiché non sono disponibili farmaci antivirali con provata efficacia clinica verso $\operatorname{BKV}(14,21,23)$. Analisi condotte nell'ultimo decennio indicano come il trattamento della malattia conclamata sia associato a un outcome subottimale del trapianto.

I progressi nello sviluppo di strumenti diagnostici e una maggior consapevolezza dellimportanza dello screening hanno portato a un trattamento della patologia nelle fasi precoci, prima che si verifichi un significativo deterioramento della funzionalità renale: questo ha comportato un miglioramento dell'outcome $(14,15$,
$21,22,24)$. Nei riceventi un trapianto renale a rischio di BKVAN, alti livelli di viruria possono essere seguiti da viremia e disfunzione renale $(8,18)$. La diagnosi di BKVAN richiede l'esecuzione di una biopsia renale, con la dimostrazione di tipiche alterazioni citopatiche da polyomavirus e presenza di nefropatia interstiziale (14, $18,21)$. Tuttavia, data la natura focale della malattia e la possibile sovrapposizione con altre patologie che complicano il decorso post-trapianto, può risultare difficile raggiungere una diagnosi precoce. $\dot{E}$ stato dimostrato che, in media, la viruria precede di 4 settimane la viremia e la viremia precede di 8 settimane la BKVAN (25). Pertanto, il monitoraggio della viruria, generalmente effettuato attraverso metodiche di citologia urinaria o PCR quantitativa, e l'analisi del DNA virale plasmatico mediante PCR quantitativa, permettono l'identificazione dei pazienti a rischio di sviluppare BKVAN. La viruria e la viremia sono associate, ma le urine e il plasma sono compartimenti separati, con la sola viremia direttamente associata alla replicazione a livello del trapianto (19). Un risultato negativo per viruria esclude una BKVAN, e può quindi essere utilmente impiegato quale test di screening di prima linea, ma la presenza di replicazione virale persistente, valutata come carica plasmatica superiore a un valore soglia (nel nostro Centro: $10^{4}$ copie/ $\mathrm{mL}$ ) mediante PCR quantitativa, è il test maggiormente predittivo per la presenza di una BKVAN “presunta” (14, $18,20,25)$. Studi prospettici condotti sia in pazienti adulti che pediatrici hanno dimostrato la fattibilità di prevenire la BKVAN, e quindi danni al trapianto, effettuando un trattamento preemptive, sulla base del monitoraggio del DNA, mediante riduzione dell'immunosoppressione (25-27).

Lo screening dell'infezione da BKV è essenziale per ottenere una diagnosi precoce e facilitare un intervento terapeutico tempestivo. La metodologia e la tempistica dovrebbero essere centro-specifiche, ed essere funzione delle competenze locali e delle caratteristiche della coorte monitorata. $\grave{E}$ stato suggerito di effettuare uno screening per la replicazione virale almeno una volta ogni 3 mesi nel primo anno, ogni sei mesi fino a 2 anni dopo il trapianto e successivamente ogni anno per 5 anni (28), o in caso di alterata funzionalità renale, o dopo il trattamento per un rigetto acuto (24). I dati ottenuti nelle coorti di età pediatrica suggeriscono che la riattivazione del BKV si verifica più precocemente nei bambini che negli adulti (27): si consiglia quindi di eseguire il monitoraggio dei riceventi pediatrici mensilmente nei primi tre mesi. In presenza di viremia, la carica virale plasmatica dovrebbe essere rivalutata ad intervalli di 2-3 settimane, per stabilirne la cinetica. La conferma o l'aumento del BKV DNA al di sopra del valore soglia 
richiedono un intervento terapeutico, che può essere guidato dalla funzionalità renale dei pazienti. I dati ottenuti in uno studio prospettico indicano che l'incidenza della viremia è più elevata nei pazienti pediatrici (27), ma l'outcome sembra essere più favorevole, in quanto la prevalenza di BKVAN e il tasso di perdita del trapianto sono risultati simili a quelli osservati negli adulti $(14,23$, 29). Per quanto esposto, nei bambini asintomatici con una carica virale superiore alla soglia stabilita, può essere ragionevole adottare un approccio d'intervento cauto, e prendere in considerazione una riduzione terapeutica dell'immunosoppressione solo in presenza di viremia persistente. In caso di disfunzione renale, deve essere eseguita una biopsia del rene trapiantato, e il trattamento deve essere somministrato in base ai risultati istologici della biopsia. In particolare, se si è escluso un rigetto acuto concomitante, può essere avviata una riduzione terapeutica dell'immunosoppressione. In caso contrario, si consiglia di trattare in primo luogo il rigetto, e quindi procedere con il trattamento della BKVAN: a quest'ultimo si dovrebbe ricorrere anche in caso di BKVAN presunta.

Per trattare una BKVAN "presunta", sono state proposte differenti strategie di riduzione della terapia immunosoppressiva, che si sono dimostrate efficaci nell'indurre clearance della viremia e nel prevenire l'insorgenza di danni renali $(14,22,25-27,30)$. In particolare, il nostro ed altri gruppi hanno privilegiato la riduzione degli inibitori della calcineurina (CNI) in una prima fase terapeutica, seguita dalla riduzione/sospensione degli antimetaboliti $(14,15,23,27)$. Nella popolazione pediatrica, la riduzione terapeutica dei CNI dovrebbe essere fatta lentamente, iniziando con una riduzione del 15-20\% (27). In alternativa, può essere effettuato lo switch dal Tacrolimus alla Ciclosporina A, oppure da un CNI a un inibitore dell'mTOR $(14,23)$.

\section{Epstein-Barr virus}

La malattia linfoproliferativa post-trapianto (PTLD) è una complicanza che si riscontra nell'1-9\% dei riceventi un trapianto renale $(31,32)$. Diversi fattori aumentano notevolmente il rischio di sviluppare una PTLD: l'infezione da virus di Epstein-Barr (EBV) è un fattore critico nella patogenesi della maggior parte dei casi osservati. Nei soggetti sani sieropositivi, esiste un equilibrio molto delicato tra i linfociti B infettati dall'EBV e la risposta immune cellulare anti-EBV, principalmente linfociti $\mathrm{T}$ citotossici CD8+ EBV-specifici (EBV-CTL) (33). Quindi, il grado di immunosoppressione farmacologica, e/o l'incompatibilità HLA e l'assenza di un numero protettivo di linfociti T, sono i principali fattori di rischio per lo sviluppo di una PTLD (34). La più alta incidenza di PTLD si osserva nei bambini (35), in quanto due dei principali fattori di rischio, ovvero la sieronegatività per EBV e l'assunzione di un elevato carico di terapia immunosoppressiva, sono generalmente prerogativa peculiare di questa coorte.

L'Organizzazione Mondiale della Sanità (WHO) ha classificato i disordini linfoproliferativi post-trapianto in quattro categorie: lesioni precoci; PTLD polimorfa, PTLD monomorfa e PTLD tipo linfoma di Hodgkin (36). Le PTLD monomorfe sono simili ai linfomi osservati in pazienti non trapiantati, in maggior parte linfomi a cellule $\mathrm{B}$, anche se possono verificarsi casi di linfomi a cellule $\mathrm{T} / \mathrm{NK}$, o forme plasmacellulari simili al mieloma. Nei riceventi un trapianto di organo solido (SOT), fino a un terzo dei casi sono risultati EBV-negativi all'analisi istologica, soprattutto tra le PTLD ad esordio tardivo (35-38). La maggior parte delle PTLD insorte dopo SOT originano dal ricevente, mentre la fonte dell'EBV può essere il donatore, il ricevente o un'infezione primaria contratta per via orale.

La diagnosi di malattia da EBV è inizialmente suggerita dalla storia clinica e dall'esame obiettivo, in associazione all'esecuzione di esami di imaging. Il decorso clinico della PTLD dopo un SOT è eterogeneo. I pazienti si presentano tipicamente con adenopatie periferiche, epatosplenomegalia e/o tonsillite, mentre una storia di diarrea può essere indicativa di malattia gastrointesinale $\mathrm{e}$ nei trapiantati di rene può essere interessato il trapianto stesso. Raramente, pazienti profondamente immunodepressi possono sviluppare una malattia fulminante con coinvolgimento multiorgano. Tuttavia, i sintomi clinici possono essere scarsi e la diagnosi di PTLD EBV-correlata deve essere considerata in tutti i pazienti trapiantati a rischio con febbre di origine sconosciuta che dura da qualche giorno. Per la diagnosi di PTLD è necessaria la conferma istologica su prelievo bioptico, anche se nelle semplici sindromi febbrili può non essere presente uno specifico coinvolgimento tissutale e, in alcuni pazienti, le lesioni possono essere inaccessibili alla biopsia.

L'insorgenza della PTLD è preceduta da una fase preclinica caratterizzata da un elevata carica di EBV nel sangue periferico, il monitoraggio dei livelli di EBV-DNA nel sangue mediante PCR rappresenta un utile strumento per ottenere una diagnosi precoce ed intraprendere un trattamento tempestivo $(7,39-45)$. Inoltre, poiché il successo della terapia è associato alla scomparsa dell'EBVDNA dal sangue, la valutazione della carica virale è utile per monitorare la risposta al trattamento $(7,40-41)$. ̀̀ stato, tuttavia, dimostrato come la clearance del DNA virale dal sangue periferico, in caso di trattamento con 
l'anticorpo monoclonale anti-CD20 Rituximab, può nascondere in realtà una malattia persistente (40). $\mathrm{Al}$ contrario, livelli persistentemente alti di EBV-DNA, soprattutto in assenza di una risposta clinica al trattamento, suggeriscono la necessità di modificare l'approccio terapeutico alla PTLD.

Il trattamento della PTLD EBV-correlata si basa sulla riduzione della massa tumorale con farmaci citotossici $(35,46,47)$ e/o con anticorpi monoclonali diretti contro i linfociti B $(40,46,48)$, e sul ripristino dell'immunità virus specifica, attraverso una riduzione dell'immunosoppressione farmacologica (24) o somministrando linfociti T citotossici EBV-specifici (33, $41,46)$. Nei pazienti riceventi un trapianto di organo solido, la riduzione/sospensione dell'immunosoppressione rimane il gold standard per la terapia di prima linea della PTLD, anche se vi è una grande variabilità delle percentuali di risposta nei dati riportati e le PTLD monoclonali hanno meno probabilità di risposta (31). Come effetto collaterale di questo approccio terapeutico, un aumento non specifico della risposta immune, indotto dalla riduzione dell'immunosoppressione, aumenta il rischio di sviluppare un rigetto. Il ruolo dell'interferone, delle immunoglobuline endovena e dei farmaci antivirali, anche preceduti da un trattamento sensibilizzante con l'acido grasso a catena corta arginina butirrato (49), è controverso (31, 46). La chemioterapia citotossica, basata su regimi a più farmaci convenzionalmente utilizzati per trattare linfomi de novo, è associata a elevati tassi di risposta, ma anche a una elevata tossicità farmaco indotta e a una aumentata suscettibilità alle infezioni $(46,47)$, mentre la monoterapia con Rituximab ha mostrato un buon profilo di tossicità, ma un tasso di risposta inferiore (48). L'outcome complessivo delle PTLD in pazienti riceventi un trapianto di organo solido trattati con terapia convenzionale è ancora subottimale, anche se risultati preliminari incoraggianti, in termini di tasso di remissione completa stabile, sono stati recentemente ottenuti in coorti pediatriche, usando regimi polichemioterapici a dosaggio ridotto, in combinazione con terapie biologiche quali l'infusione di EBV-CTL autologhi o allogenici $(41,46,50)$.

Il ruolo della profilassi antivirale per prevenire l'infezione da EBV, e quindi il rischio d'insorgenza della PTLD, nei riceventi un SOT è controversa (31). Nei trapiantati asintomatici, l'EBV-DNA identifica i pazienti ad alto rischio di sviluppare una PTLD (39, 42, 43), che potrebbero trarre beneficio da un approccio terapeutico preventivo, anche se la correlazione tra un'alta carica virale e l'insorgenza della PTLD dopo SOT non è così lineare come quella osservata nei ri- ceventi un trapianto di cellule staminali emopoietiche. I riceventi un trapianto di organo solido possono presentare un'alta carica virale per parecchi mesi senza sviluppare una PTLD, e non tutti i casi sono associati ad elevati livelli di EBV-DNA (43).

Nei pazienti ad alto rischio, quali i soggetti EBV-sieronegativi al momento del trapianto, si raccomanda un monitoraggio frequente, ed un eventuale approccio preemptive, per prevenire la progressione verso la PTLD (24). Allo stato attuale, mancano dati sull'efficacia dei farmaci antivirali in protocolli preemptive, mentre è stato dimostrato come la riduzione della terapia immunosoppressiva nei pazienti pediatrici riceventi un trapianto di fegato ed EBV DNA-positivi, possa prevenire lo sviluppo di PTLD (44). Sebbene la riduzione dell'immunosoppressione possa essere una misura efficace per indurre lo sviluppo di immunità specifica nei pazienti EBV-sieronegativi che contraggono una infezione primaria dopo il trapianto, i dati preliminari ottenuti dal nostro gruppo indicano come i riceventi pediatrici di rene con una carica virale persistentemente elevata, soprattutto tardivamente dopo il trapianto, non sembrino beneficiare della riduzione dell'immunosoppressione (45). In questi soggetti, al momento si consiglia uno stretto monitoraggio clinico e una terapia precoce della PTLD conclamata (41).

\section{Monitoraggio dell'immunità virus-specifica}

La quantificazione della risposta immunitaria specifi$\mathrm{ca}$, in associazione con la determinazione della carica virale, si è rivelata utile sia nella caratterizzazione dei sottogruppi di pazienti ad alto rischio per lo sviluppo di malattia $(9,51,52)$, che nella valutazione della risposta alla terapia $(41,53)$.

Nei riceventi un trapianto di fegato EBV-sieronegativi che hanno sviluppato una PTLD, è stato dimostrato come sia possibile identificare il rischio di sviluppare una malattia virus-correlata mediante analisi combinata della carica virale e della frequenza di linfociti $T$ virus-specifici (9).

Nel contesto di un'infezione da CMV, l'incapacità di controllare la replicazione virale è associata a una risposta subottimale delle cellule $\mathrm{CD} 4+$ e $\mathrm{CD} 8+$, che si presentano in basso numero e con funzione compromessa $(54,55)$. Analogamente a quanto osservato per l'EBV e il CMV, lo sviluppo di viruria, viremia e BKVAN è stato associato a una compromissione della risposta $T$ cellulare $(10,52,56)$. Inoltre, la clearance della viremia e la prevenzione della BKVAN dipendono dal recupero di una immunità $\mathrm{T}$ cellulare $\mathrm{BK}$ - 
specifica $(27,57)$. Sembra inoltre ragionevole gestire la modulazione terapeutica dell'immunosoppressione associando alla quantificazione della carica virale una valutazione dell'immunità BKV-specifica, dal momento che dati preliminari indicano come la comparsa di linfociti $\mathrm{T}$ BKV-specifici coincida con la riduzione della viremia e con una migliorata o stabile funzionalità del trapianto.

Infine, avendo dimostrato come il controllo dell'infezione dipenda dal ripristino di una risposta immunitaria protettiva, la caratterizzazione dell'immunità virus-specifica ha permesso lo sviluppo di strategie terapeutiche basate sulla somministrazione di linfociti $T$ antigeni-specifici espansi in vitro dal pool di linfociti memoria (58).

\section{Conclusioni}

L'attuazione di strategie di monitoraggio e l'applicazione di un trattamento preemptive hanno profondamente cambiato il corso delle infezioni virali dopo trapianto. L'individualizzazione della gestione del paziente, attraverso nuovi approcci che tengano conto della cinetica di replicazione virale, sarà impiegata sempre più frequentemente nei riceventi un trapianto, e, in associazione alla valutazione della funzione immunitaria, potrà offrire una strategia ottimale per il controllo delle infezioni post-trapianto. Studi prospettici di sorveglianza combinata immunologica e virale consentiranno di valutare il potenziale di questa strategia e identificare i parametri più idonei ai fini del monitoraggio.

\section{Indirizzo degli Autori:}

Fabrizio Ginevri, MD

U.O.S. Medicina Clinica e Sperimentale

dei Trapianti d'Organo

Unità Operativa di Nefrologia, Istituto G. Gaslini

Largo Gaslini 5

16147 Genova

fabrizioginevri@ospedale-gaslini.ge.it

\section{Bibliografia}

1. Fishman JA, Rubin RH. Infection in organ-transplant recipients. N Engl J Med 1998; 338: 1741-51.

2. Dharnidharka VR, Stablein DM, Harmon WE. Post-transplant infections now exceed acute rejection as cause for hospitalization: a report of the NAPRTCS. Am J Transplant 2004; 4: 384-89.

3. Hirsch HH. BK virus: opportunity makes a pathogen. Clin Infect Dis 2005; 41: 354-60.

4. Fischer SA. Emerging viruses in transplantation: there is more to infection after transplant than CMV and EBV. Transplantation 2008; 86: 1327-39.

5. Humar A, Michaels M. American Society of Transplantation recommendations for screening, monitoring and reporting of infectious complications in immunosuppression trials in recipients of organ transplantation. Am J Transplant 2006; 6: 262.

6. Kotton CN, Kumar D, Caliendo AM, et al. Transplantation Society International CMV Consensus Group. International consensus guidelines on the management of cytomegalovirus in solid organ transplantation. Transplantation 2010; 89: 779-95.

7. Baldanti F, Gatti M, Furione M, et al. Kinetics of EpsteinBarr virus DNA load in different blood compartments of pediatric recipients of T-cell depleted HLA-haploidentical stem cell transplantation. J Clin Microbiol 2008; 46: 3672-77.

8. Nickeleit V, Klimkait T, Binet IF et al. Testing for polyomavirus type BK DNA in plasma to identify renal-allograft recipients with viral nephropathy. N Engl J Med 2000; 342: 1309-15.

9. Smets F, Latinne D, Bazin H, et al. Ratio between EpsteinBarr viral load and anti-Epstein-Barr virus specific T-cell response as a predictive marker of posttransplant lymphoproliferative disease. Transplantation 2002; 73: 1603-10.

10. Ginevri F, Hirsch HH, Comoli P. Cellular immune responses to BK virus. Curr Opin Organ Transplant 2008; 13: 569-74.

11. Rooney CM, Smith CA, Ng CY, et al. Use of gene-modified virus-specific T lymphocytes to control Epstein-Barrvirus-related lymphoproliferation. Lancet 1995; 345: 9-12.

12. Choquet S, Leblond V, Herbrecht R, et al. Efficacy and safety of rituximab in B-cell post-transplantation lymphoproliferative disorders: results of a prospective multicenter phase 2 study. Blood 2006; 107: 3053-57.

13. Paya C, Humar A, Dominguez E, et al. Valganciclovir Solid Organ Transplant Study Group Efficacy and safety of valganciclovir vs. oral ganciclovir for prevention of cytomegalovirus disease in solid organ transplant recipients. Am J Transplant 2004; 4: 611-20.

14. Hirsch HH, Brennan DC, Drachenberg CB, et al. Polyomavirus-associated nephropathy in renal transplantation: 
interdisciplinary analyses and recommendations. Transplantation 2005; 79: 1277-86.

15. Vasudev B, Hariharan S, Hussain SA, Zhu YR, Bresnahan BA, Cohen EP. BK virus nephritis: risk factors, timing, and outcome in renal transplant recipients. Kidney Int 2005; 68: 1834-9.

16. Ramos E, Drachenberg CB, Papadimitriou JC, et al. Clinical course of polyoma virus nephropathy in 67 renal transplant patients. J Am Soc Nephrol 2002; 13: 2145-51.

17. Schold JD, Rehman S, Kayle LK, Magliocca J, Srinivas TR, Meier-Kriesche HU. Treatment for BK virus: incidence, risk factors and outcomes for kidney transplant recipients in the United States. Transpl Int 2009; 22: 626-34.

18. Hirsch HH, Steiger J. Polyomavirus BK. Lancet Infect Dis 2003; 3: 611-23.

19. Funk GA, Steiger J, Hirsch HH. Rapid dynamics of polyomavirus type BK in renal transplant recipients. J Infect Dis 2006; 193: 80-7.

20. Randhawa PS, Finkelstein S, Scantlebury V, et al. Human Polyoma virus-associated interstial nephritis in the allograft kidney. Transplantation 1999; 67: 103.

21. Drachenberg CB, Papadimitriou JC, Hirsch HH, et al. Histological patterns of polyomavirus nephropathy: Correlation with graft outcome and viral load. Am J Transplantation 2004; 4: 2082- 92.

22. Ginevri F, Hirsch HH. Polyomavirus-associated nephropathy. In: Molony DA, Craig JC eds. Evidence-Based Nephrology, Oxford, UK: Wiley-Blackwell, 2008.

23. Acott PD, Hirsch HH. BK virus infection, replication, and diseases in pediatric kidney transplantation. Pediatr Nephrol 2007; 22: 1243-50.

24. KDIGO Guidelines. Am J Transplant 2009; 9 (Suppl 3): S44-58.

25. Hirsch HH, Knowles W, Dickenmann M, et al. Prospective study of polyomavirus type BK replication and nephropathy in renal-transplant recipients. N Engl J Med 2002; 347: 488-96.

26. Brennan DC, Agha I, Bohl DL, et al. Incidence of BK with tacrolimus versus cyclosporine and impact of preemptive immunosuppression reduction. Am J Transplant 2005; 5: $582-94$.

27. Ginevri F, Azzi A, Hirsch HH, et al. Prospective monitoring of polyomavirus BK replication and impact of preemptive intervention in pediatric kidney recipients. Am J Transplant 2007; 7: 2727-35.

28. Hirsch HH Randhawa P, AST Infectious Diseases Community of Practice. BK virus in solid organ transplant recipients. Am J Transplant 2009; 9 (Suppl 4): S136-46.

29. Saad ER, Bresnahan BA, Cohen EP, et al. Successful treatment of BK viremia using reduction in immunosuppression without antiviral therapy. Transplantation 2008; 85 : 850-4.
30. Ginevri F, De Santis R, Comoli P, et al. Polyomavirus BK infection in pediatric kidney-allograft recipients: a singlecenter analysis of incidence, risk factors, and novel therapeutic approaches. Transplantation 2003; 75: 1266-70.

31. Green M. Management of Epstein-Barr virus-induced post-transplant lymphoproliferative disease in recipients of solid organ transplantation. Am J Transplant 2001; 1 : 103-8.

32. Opelz G, Dohler B. Lymphomas after solid organ transplantation: a collaborative transplant study report. Am J Transplant 2003; 4: 222-30.

33. Merlo A, Turrini R, Dolcetti R, et al. The interplay between EBV and the immune system: a rationale for adoptive cell therapy of EBV-related disorders. Haematologica 2010; 95: 1769-77.

34. Dharnidharka VR, Harmon WE. Management of pediatric postrenal transplantation infections. Semin Nephrol 2001; 21: 521-31.

35. Gross TG, Steinbuch M, DeFor T, et al. B cell lymphoproliferative disorders following hematopoietic stem cell transplantation: risk factors, treatment and outcome. Bone Marrow Transplant 1999; 23: 251-8.

36. Swerdlow SH, Campo E, Harris NL, et al. WHO Classification of Tumours of Haematopoietic and Lymphoid Tissues (ed 4th) 2008. Lyon, France: IARC Press.

37. Cohen JI. Epstein-Barr virus infection. N Engl J Med 2000; 343: 481-92.

38. Nalesnik M, Jaffe R, Starzl TE, et al. The pathology of posttransplant lymphoproliferative disorders occurring in the setting of cyclosporin A-prednisone immunosuppression. Am J Pathol 1988; 133: 173-92.

39. Baldanti F, Grossi P, Furione M, et al. High levels of Epstein-Barr virus DNA in blood of solid-organ transplant recipients and their value in predicting posttransplant lymphoproliferative disorders. J Clin Microbiol 2000; 38: 613-9.

40. Yang J, Tao Q, Flinn IW, et al. Characterization of EpsteinBarr virus-infected $B$ cells in patients with posttransplantation lymphoproliferative disease: disappearance after rituximab therapy does not predict clinical response. Blood 2000; 96: 4055-63.

41. Comoli P, Maccario R, Locatelli F, et al. Treatment of EBV-related post-renal transplant lymphoproliferative disease with a tailored regimen including EBV-specific T cells. Am J Transplant 2005; 5: 1415-22.

42. Savoie A, Perpete C, Carpentier L, Joncas J, Alfieri C. Direct correlation between the load of Epstein-Barr virusinfected lymphocytes in the peripheral blood of pediatric transplant patients and risk of lymphoproliferative disease. Blood 1994; 83: 2715-22.

43. Rowe DT, Webber S, Schauer EM, Reyes J, Green M. Epstein-Barr virus load monitoring: its role in the prevention 
and management of post-transplant lymphoproliferative disease. Transpl Infect Dis 2001; 3: 79-87.

44. Lee TC, Savoldo B, Rooney CM, et al. Quantitative EBV viral loads and immunosuppression alterations can decrease PTLD incidence in pediatric liver transplant recipients. Am J Transplant 2005; 5: 2222-8.

45. Ginevri F, Di Marco E, Parodi A, et al.. EBV viral load monitoring and reduction of immunosuppression do not successfully prevent PTLD after pediatric kidney transplantation. Am J Transplant 2008; 8(s2): 477.

46. Comoli P, Rooney CM. Treatment of Epstein-Barr virus infections. In: Jenson HB, Tselis A eds. Epstein-Barr virus, New York: Taylor \& Francis, 2006; 351-72.

47. Swinnen LJ, Mullen GM, Carr TJ, Costanzo MR, Fisher RI. Aggressive treatment for postcardiac transplant lymphoproliferation. Blood 1995; 86: 3333-40.

48. Choquet S, Leblond V, Herbrecht R, et al. Efficacy and safety of rituximab in B-cell post-transplantation lymphoproliferative disorders: results of a prospective multicenter phase 2 study. Blood 2006; 107: 3053-7.

49. Perrine SP, Hermine O, Small T, et al. A phase $1 / 2$ trial of arginine butyrate and ganciclovir in patients with EpsteinBarr virus-associated lymphoid malignancies. Blood 2007; 109: 2571-8.

50. Gross TG, Bucuvalas JC, Park JR et al. Low-dose chemotherapy for Epstein-Barr virus-positive post-transplantation lymphoproliferative disease in children after solid organ transplantation. J Clin Oncol 2005; 23: 6481-8.

51. Yang J, Lemas VM, Flinn IW, Krone C, Ambinder RF. Ap- plication of the ELISPOT assay to the characterization of CD8+ responses to Epstein-Barr virus antigens. Blood 2000; 95: 241-8.

52. Comoli P, Azzi A, Maccario R, et al. Polyomavirus BK-specific immunity after kidney transplantation. Transplantation 2004; 78: 1229-32.

53. Savoldo B, Rooney CM, Quiros-Tejeira RE, et al. Cellular immunity to Epstein-Barr virus in liver transplant recipients treated with rituximab for post-transplant lymphoproliferative disease. Am J Transplant 2005; 5: 566-72.

54. Mattes FM, Vargas A, Kopycinski J, et al. Functional Impairment of Cytomegalovirus Specific CD8 T Cells Predicts High-Level Replication After Renal Transplantation. Am J Transplant 2008; 8: 990-9.

55. Sester U, Presser D, Dirks J, Gartner BC, Kohler H, Sester M. PD-1 expression and IL-2 loss of cytomegalovirus- specific $T$ cells correlates with viremia and reversible functional anergy. Am J Transplant 2008; 8: 1486-97.

56. Binggeli S, Egli A, Schaub S, et al. Polyomavirus BKspecific cellular immune response to VP1 and Large Tantigen in kidney transplant recipients. Am J Transplant 2007; 7: 1131-9.

57. Prosser SE, Orentas RJ, Jurgens L, Cohen EP, Hariharan S. Recovery of BK virus Large-T-antigen-specific cellular immune response correlates with resolution of BK virus nephropathy. Transplantation 2008; 85: 185-92.

58. Comoli P, Locatelli F, Ginevri F, Maccario R. Cellular immunotherapy for viral infections in solid organ transplant recipients. Curr Opin Organ Transplant 2002; 7: 314-9. 